\title{
To Explore the Potential Mechanism of Yiqing Capsule in the Treatment of COVID-19 Based on Network Pharmacology
}

\author{
Ying Wang1, Jingyi Zhao², Yinhui Yao', Song Jiang1, Xinhong Zhao', Junhui Hu1* \\ ${ }^{1}$ Department of Pharmacy, The Affiliated Hospital of Chengde Medical College, Chengde, China \\ ${ }^{2}$ Department of Functional Center, Chengde Medical College, Chengde, China \\ Email: ^hujhcyfy@163.com
}

How to cite this paper: Wang, Y., Zhao, J.Y., Yao, Y.H., Jiang, S., Zhao, X.H. and Hu, J.H. (2020) To Explore the Potential Mechanism of Yiqing Capsule in the Treatment of COVID-19 Based on Network Pharmacology. Chinese Medicine, 11, 96-104. https://doi.org/10.4236/cm.2020.112005

Received: April 25, 2020

Accepted: June 5, 2020

Published: June 8, 2020

Copyright $\odot 2020$ by author(s) and Scientific Research Publishing Inc. This work is licensed under the Creative Commons Attribution International License (CC BY 4.0).

http://creativecommons.org/licenses/by/4.0/

\begin{abstract}
Objective: To select the ingredients and targets of Yiqing capsule in treating COVID-19 based on network pharmacology, then to explore the potential mechanism of Yiqing capsule in treating COVID-19. Methods: We screened the ingredients and targets of Yiqing capsule on Traditional Chinese Medicine Systems Pharmacology Database and Analysis Platform (TCMSP), and target genes for COVID-19 in GeneCards database, constructed drug-ingredient-target network through software Cytoscape 3.7.2, and constructed protein protein interaction network (PPI) network through STRING database. Gene Ontology (GO) enrichment analysis and Kyoto Encyclopedia of Genes and Genomes (KEGG) analysis of key gene targets of Yiqing capsule are used to treat COVID-19 through software R5.3.2. Results: We got 42 ingredients, 42 potential therapeutic targets, 1643 GO items and 970 pathways in our study. The main pathway including IL-17 signaling pathway, Chagas disease (American trypanosomiasis), Influenza $\mathrm{A}$, and TNF signaling pathway. Conclusion: Yiqing capsule plays a role in treating COVID-19 through multiple ingredients, multiple targets and multiple pathways.
\end{abstract}

\section{Keywords}

Yiqing Capsule, Network Pharmacology, COVID-19

\section{Introduction}

Coronavirus disease 2019 (COVID-19) was named by the WHO on January 12, 2020, and the pathogen was named SARS-CoV-2 [1]. COVID-19 is caused by a novel, highly infectious coronavirus resulting in a pandemic. The main clinical symptoms are fever and dry cough. A few are presented with gastrointestinal 
symptoms, severe respiratory failure [2]. COVID-19 is considered to be a blight in traditional Chinese medicine (TCM) [3]. The third edition of "diagnosis and treatment scheme of COVID-19" published by the government of China (GOC) recommended Chinese medicine therapy in the treatment in COVID-19, achieving good results in prophylaxis and treatment. Research on mining the regularity of Chinese medicine prescription in treating COVID-19 had also been shown that antipyretic and cathartic (qing re xie xia) drugs are used throughout all the stages of anti-COVID-19 [4].

The formula of Yiqing capsule is the representative prescription of fire cathartic. It was derived from the "Xie Xin Tang" in "Jin Kui Yao Lue" written by Zhang Zhongjing in Han dynasty. The Yiqing capsule has the function of purging fire and detoxification, removing phlegm and hemostasis, transforming dampness into clear heat. Yiqing capsule exists in 3 TCM systems: Radix Rhei Et Rhizome (Dahuang), Coptidis Rhizoma (Huanglian), and Scutellariae Radix (Huangqin) [5]. Yiqing capsule is used to treat upper respiratory tract infection, and H1N1 influenza in clinical practice [6] [7]. Therefore, our study analyzed the ingredients, targets and pathway of Yiqing capsule, to explore the possible effect and potential mechanism of Yiqing capsule in the treatment of COVID-19, and further verify the importance of fire relieving prescription in the treatment of COVID-19, in order to provide basis for further basic research and clinical application.

\section{Materials and Methods}

\subsection{Screened for Ingredients and Targets of Yiqing Capsule}

Screened for ingredients and targets of Yiqing capsule with Radix Rhei Et Rhizome (Dahuang), Coptidis Rhizoma (Huanglian), Scutellariae Radix (Huangqin) as the index words on TCMSP (http://tcmspw.com/), was performed with the screening conditions oralbioavailability $(\mathrm{OB}) \geq 30 \%$ and drug-likeness (DL) $>$ 0.18 . The target protein was then transformed into a Gene Symbol through Uniprot Database (http://www.uniprot.org/).

\subsection{Constructed Drug-Ingredient-Target Network}

Target genes for COVID-19 were searched for in the GeneCards database (http://www.genecards.org/) with the keyword "Novel Coronavirus Pneumonia", then compaired with targets of Yiqing capsule, screening the same target genes. A drug-ingredient-target network of Yiqing capsule in treating COVID-19 was constructed through software Cytoscape 3.7.2.

\subsection{Constructed PPI Network}

The potential therapeutic targets of Yiqing capsule in treatment of COVID-19 were imported into the STRING database (https://string-db.org/). The organism selected was "Homo sapiens". The minimum required interaction score was set to 0.4. Then the key targets were screened through software R5.3.2. 


\subsection{Gene Ontology (G0) and Kyoto Encyclopedia of Genes and Genomes (KEGG) Enrichment Analysis}

The GO enrichment analysis and KEGG pathway analysis of key gene targets of Yiqing capsule in treating COVID-19 were analyzed by software R5.3.2, with P $\leq$ 0.05 as screening conditions. Every item was sorted according to the number of targets involved in each item and the lgP value.

\section{Results}

\subsection{Screened the Ingredients and Targets of Yiqing Capsule}

We selected 259 targets from the GeneCards database with the key words "Novel Coronavirus Pneumonia". Furthermore, we mapped a total of 42 potential therapeutic targets in Yiqing capsule. We also screened 42 ingredients in this study. The results are shown in Table 1 and Figure 1.

Table 1. Yiqing capsule Ingredients in the treatment of COVID-19.

\begin{tabular}{|c|c|c|c|c|c|}
\hline & Mol ID & Molecule Name & MW & OB (\%) & DL \\
\hline \multicolumn{6}{|c|}{ dahuang (6 ingredients) } \\
\hline 1 & MOL000096 & $(-)$-catechin & 290.29 & 49.68 & 0.24 \\
\hline 2 & MOL000358 & beta-sitosterol & 414.79 & 36.91 & 0.75 \\
\hline 3 & MOL000471 & aloe-emodin & 270.25 & 83.38 & 0.24 \\
\hline 4 & MOL002235 & EUPATIN & 360.34 & 50.8 & 0.41 \\
\hline 5 & MOL002268 & rhein & 284.23 & 47.07 & 0.28 \\
\hline 6 & MOL002281 & Toralactone & 272.27 & 46.46 & 0.24 \\
\hline \multicolumn{6}{|c|}{ huanglian (9 ingredients) } \\
\hline 7 & MOL000098 & quercetin & 302.25 & 46.43 & 0.28 \\
\hline 8 & MOL000785 & palmatine & 352.44 & 64.6 & 0.65 \\
\hline 9 & MOL001454 & berberine & 336.39 & 36.86 & 0.78 \\
\hline 10 & MOL001458 & coptisine & 320.34 & 30.67 & 0.86 \\
\hline 11 & MOL002668 & Worenine & 334.37 & 45.83 & 0.87 \\
\hline 12 & MOL002894 & berberrubine & 322.36 & 35.74 & 0.73 \\
\hline 13 & MOL002897 & epiberberine & 336.39 & 43.09 & 0.78 \\
\hline 14 & MOL002903 & (R)-Canadine & 339.42 & 55.37 & 0.77 \\
\hline 15 & MOL002904 & Berlambine & 351.38 & 36.68 & 0.82 \\
\hline \multicolumn{6}{|c|}{ huangqin (27 ingredients) } \\
\hline 16 & MOL000073 & ent-Epicatechin & 290.29 & 48.96 & 0.24 \\
\hline 17 & MOL000173 & wogonin & 284.28 & 30.68 & 0.23 \\
\hline 18 & MOL000228 & $\begin{array}{l}\text { (2R)-7-hydroxy-5-methoxy-2-phenylchroman- } \\
\text { 4-one }\end{array}$ & 270.3 & 55.23 & 0.2 \\
\hline 19 & MOL000358 & beta-sitosterol & 414.79 & 36.91 & 0.75 \\
\hline 20 & MOL000449 & Stigmasterol & 412.77 & 43.83 & 0.76 \\
\hline
\end{tabular}




\section{Continued}

\begin{tabular}{|c|c|c|c|c|c|}
\hline 21 & MOL000525 & Norwogonin & 270.25 & 39.4 & 0.21 \\
\hline 22 & MOL000552 & 5,2'-Dihydroxy-6,7,8-trimethoxyflavone & 344.34 & 31.71 & 0.35 \\
\hline 23 & MOL001458 & coptisine & 320.34 & 30.67 & 0.86 \\
\hline 24 & MOL002714 & baicalein & 270.25 & 33.52 & 0.21 \\
\hline 25 & MOL002897 & epiberberine & 336.39 & 43.09 & 0.78 \\
\hline 26 & MOL002909 & 5,7,2,5-tetrahydroxy-8,6-dimethoxyflavone & 376.34 & 33.82 & 0.45 \\
\hline 27 & MOL002910 & Carthamidin & 288.27 & 41.15 & 0.24 \\
\hline 28 & MOL002913 & Dihydrobaicalin_qt & 272.27 & 40.04 & 0.21 \\
\hline 29 & MOL002914 & Eriodyctiol (flavanone) & 288.27 & 41.35 & 0.24 \\
\hline 30 & MOL002915 & Salvigenin & 328.34 & 49.07 & 0.33 \\
\hline 31 & MOL002917 & 5,2',6'-Trihydroxy-7,8-dimethoxyflavone & 330.31 & 45.05 & 0.33 \\
\hline 32 & MOL002925 & $5,7,2$ ',6'-Tetrahydroxyflavone & 286.25 & 37.01 & 0.24 \\
\hline 33 & MOL002927 & Skullcapflavone II & 374.37 & 69.51 & 0.44 \\
\hline 34 & MOL002928 & oroxylin a & 284.28 & 41.37 & 0.23 \\
\hline 35 & MOL002932 & Panicolin & 314.31 & 76.26 & 0.29 \\
\hline 36 & MOL002933 & 5,7,4'-Trihydroxy-8-methoxyflavone & 300.28 & 36.56 & 0.27 \\
\hline 37 & MOL002934 & NEOBAICALEIN & 374.37 & 104.34 & 0.44 \\
\hline 38 & MOL002937 & DIHYDROOROXYLIN & 286.3 & 66.06 & 0.23 \\
\hline 39 & MOL008206 & Moslosooflavone & 298.31 & 44.09 & 0.25 \\
\hline 40 & MOL012245 & 5,7,4'-trihydroxy-6-methoxyflavanone & 302.3 & 36.63 & 0.27 \\
\hline 41 & MOL012246 & 5,7,4'-trihydroxy-8-methoxyflavanone & 302.3 & 74.24 & 0.26 \\
\hline 42 & MOL012266 & rivularin & 344.34 & 37.94 & 0.37 \\
\hline
\end{tabular}

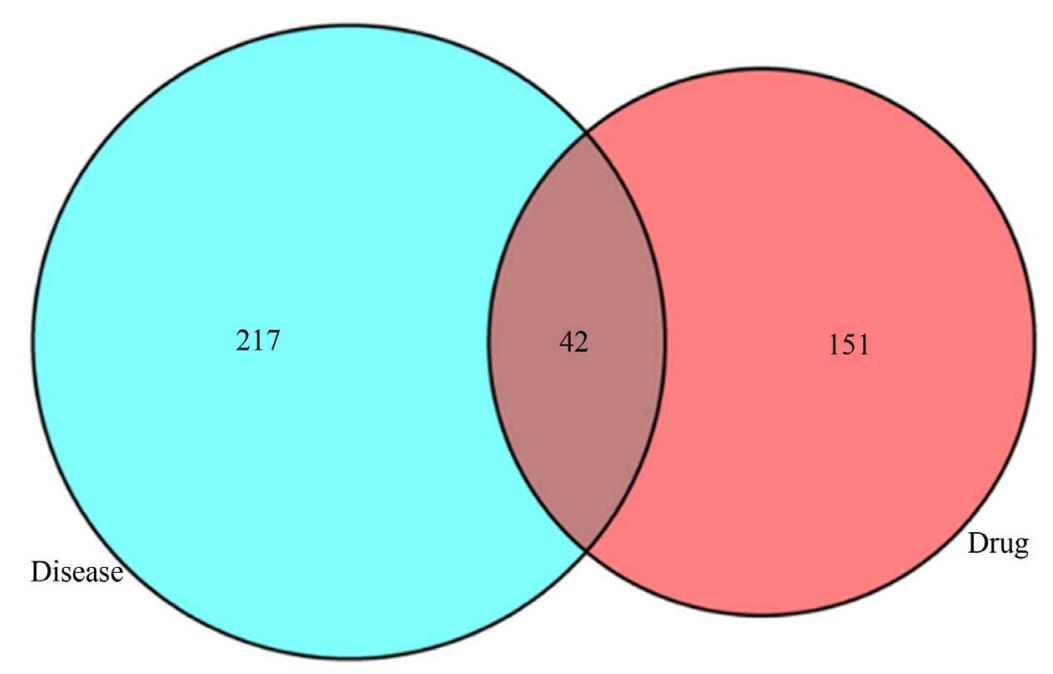

Figure 1. Veeny diagram of Yiqing capsule targets and COVID-19 targets.

\subsection{Constructed Drug-Ingredient-Target Network}

We constructed a drug-ingredient-target network of 42 selected targets of Yiqing 
capsule in treating COVID-19 through software Cytoscape (shown in Figure 2). We got 86 nodes and 263 edges. When sorted by degree, the median ingredient number was 4 , the top 6 ingredients potentially interacting with the targets were coptisine, naloe-emodin, baicalein, beta-sitosterol, wogonin, Eriodyctiol (flavanone), and oroxylin a.

\subsection{Constructed PPI Network}

The 42 potential targets of Yiqing capsule in the treatment of COVID-19 were constructed to a PPI network through STRING database (Shown in Figure 3).

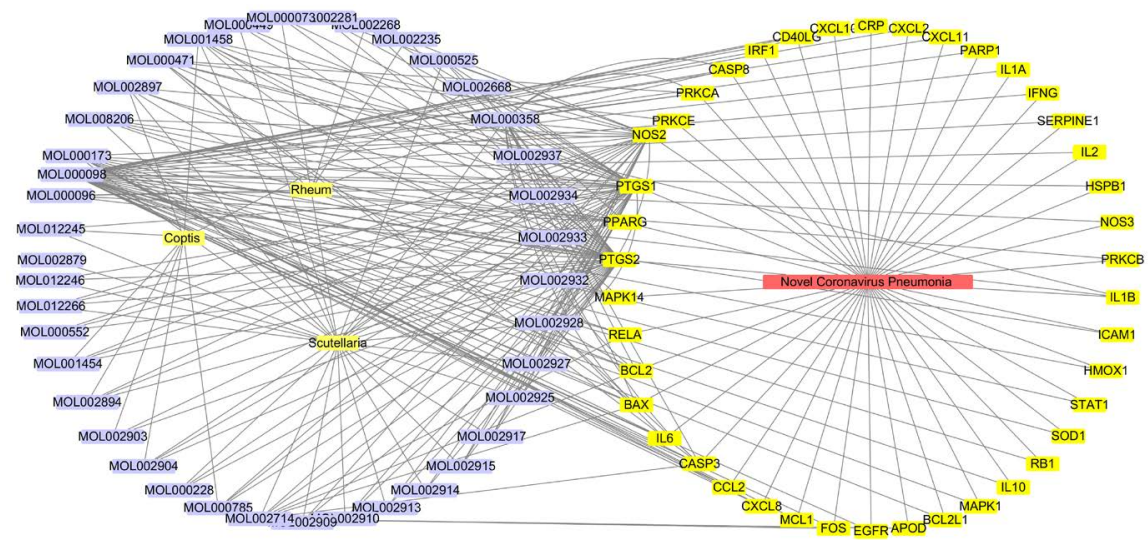

Figure 2. Medicinal material-ingredient-target-disease network. Key: yellow-TCM, purple—ingredient, green — targets, red-disease.

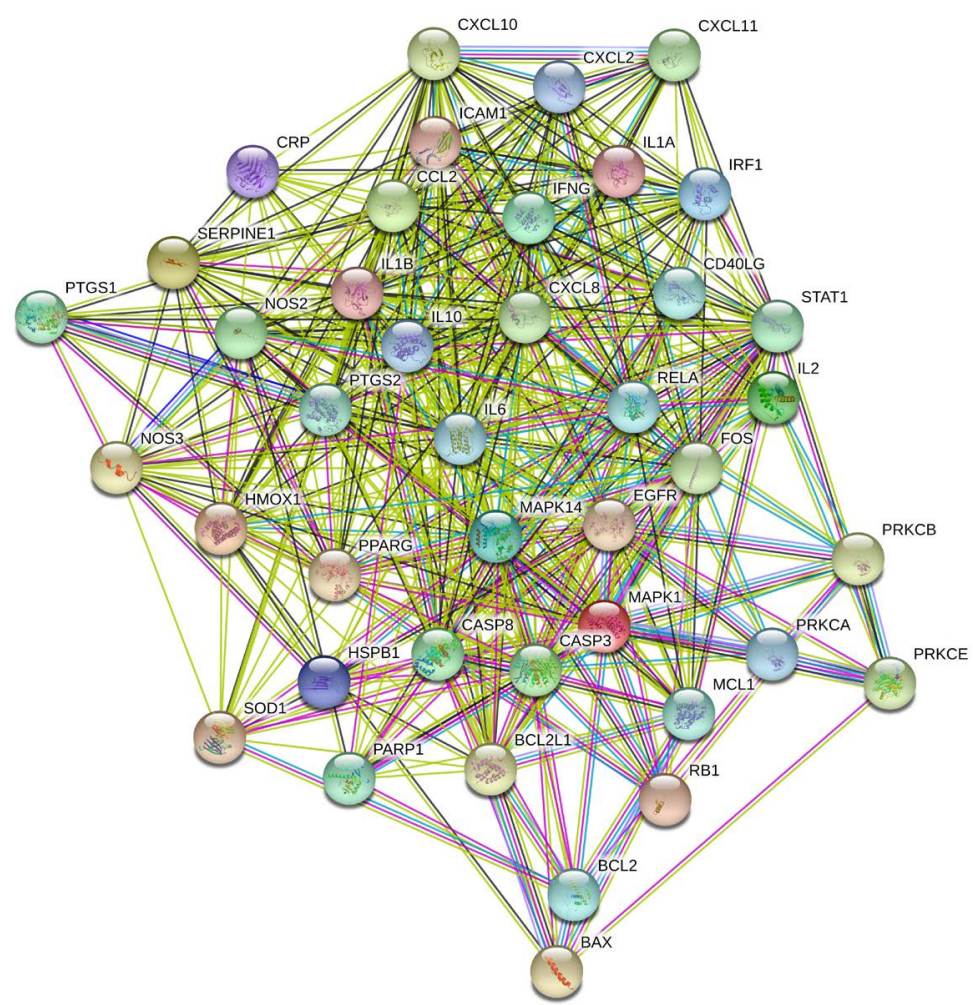

Figure 3. PPI network of Yiqing capsule in the treatment of COVID-19. 
The median was 24, the top 8 key targets were Interleukin-6 (IL6), Caspase-3 (CASP3), Interleukin-10 (IL10), Mitogen-activated protein kinase 1 (MAPK1), Interleukin-8 (CXCL8), C-C motif chemokine 2 (CCL2), Interleukin-1 beta (IL1B), Prostaglandin G/H synthase 2 (PTGS2).

\subsection{GO Nrichment Analysis and KEGG Pathway Analysis}

Forty-two potential targets were analyzed through software R, resulting in 1643 GO items (76 MF items, $15 \mathrm{CC}$ items, and $1522 \mathrm{BP}$ items), as shown in Figure 4. We got 970 pathways through KEGG pathway enrichment analysis (shown in Figure 5), such as AGE-RAGE signaling pathway in diabetic complications, IL-17 signaling pathway, Chagas disease (American trypanosomiasis), Influenza A, TNF signaling pathway, and Pertussis.

\section{Discussion}

COVID-19 is considered to be a blight in the seventh edition of "diagnosis and treatment scheme of COVID-19" published by National Health Commission of the People's Republic of China. COVID-19 is mainly caused by "Warm heat

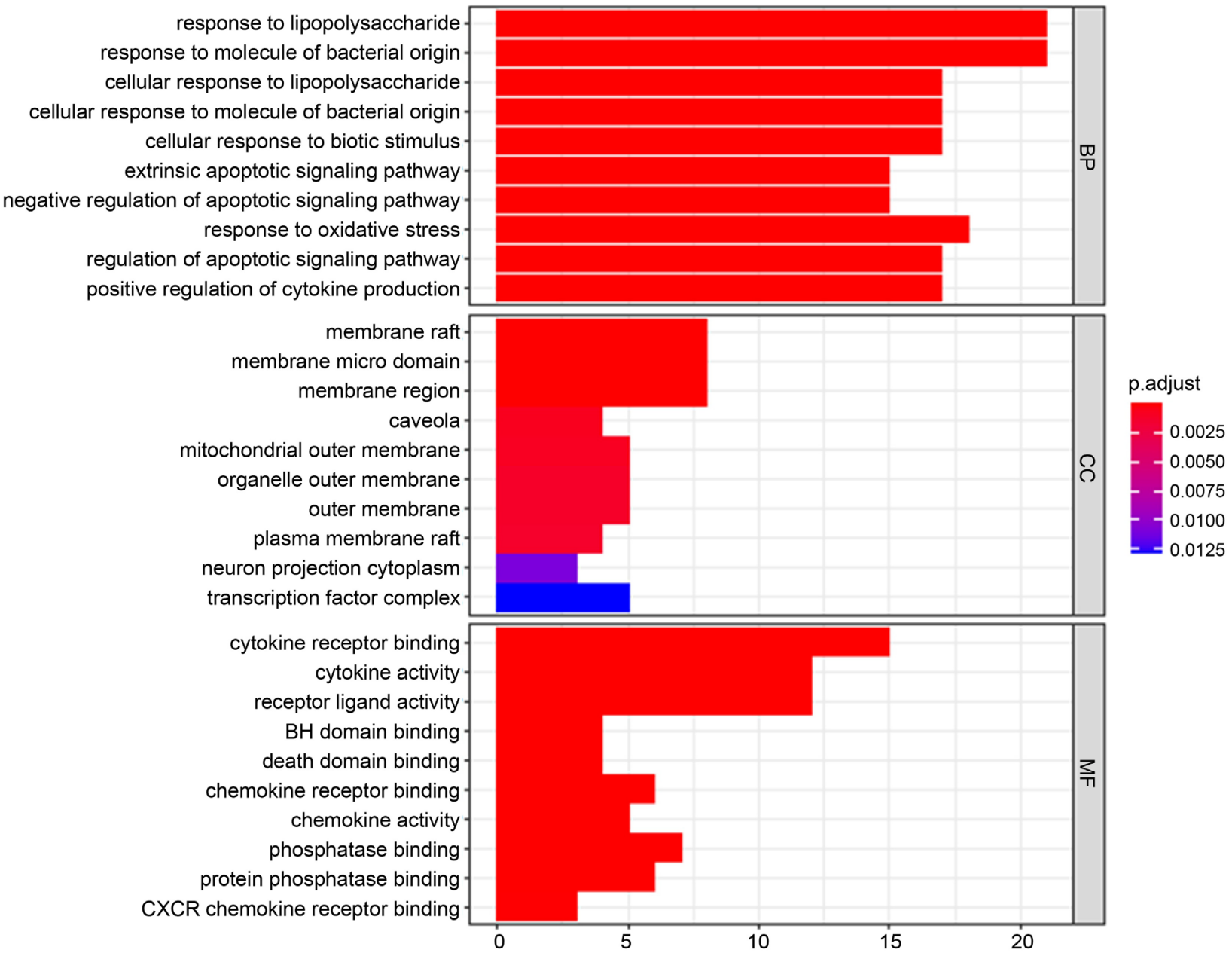

Figure 4. GO nrichment analysis of Yiqing capsule. 
AGE-RAGE signaling pathway in diabetic complications

IL-17 signaling pathway-

Chagas disease(American trypanosomiasis)

Influenza A-

TNF signaling pathway

Pertussis

Leishmaniasis -

Hepatitis B-

Amoebiasis-

Tuberculosis -

Human cytomegalovirus infection-

H IF-1 signaling pathway-

Toxoplasmosis -

Kaposi sarcoma-associated herpesvirus infection-

Measles -

NF-kappaB signaling pathway

Toll-like receptor signaling pathway-

C-type lectin receptor signaling pathway-

NOD-like receptor signaling pathway-

Fluid shear stress and atherosclerosis

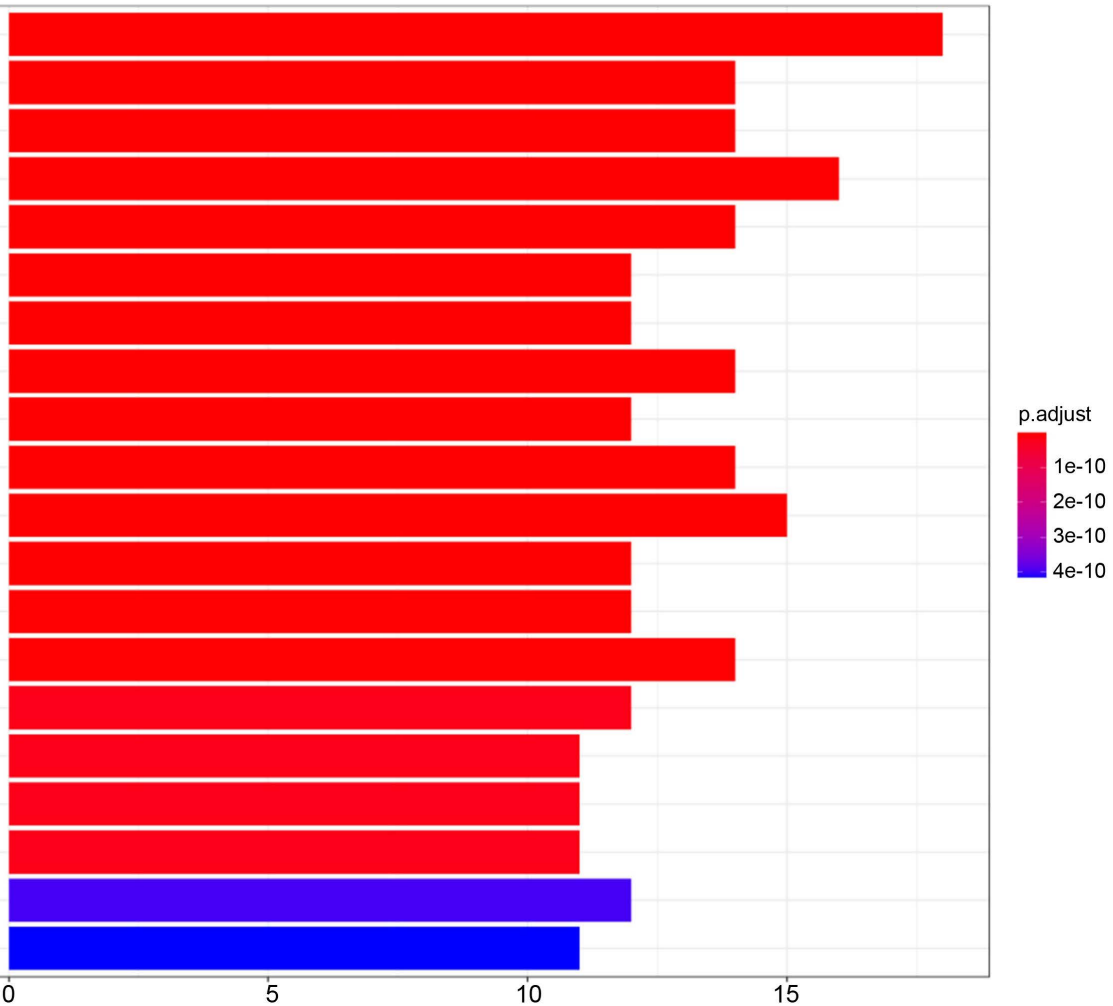

Figure 5. KEGG pathway nrichment analysis of Yiqing capsule.

toxic evil" (wen re du xie), affecting the lungs, the main syndromes in TCM include warm pathogenic factors affecting the lung, warm obstructing lung and warm poisoning blocking lungs. Most medical experts considered that the pathogenic factor of COVID-19 was "heat toxic evil blight" (Re du xie blight), allowing use of the theory of febrile diseases as clinical guidelines under different syndromes [8] [9]. A published study showed that "Qing xia" plays a key role in the diagnosis and treatment of epidemic diseases [10]. Yiqing capsule is composed of Radix Rhei Et Rhizome (Dahuang), Coptidis Rhizoma (Huanglian), and Scutellariae Radix (Huangqin). The tropism of Radix Rhei Et Rhizome (Dahuang) is bitter and cold, in stomach, liver, large intestine meridians, with functions to Discharge heat poison, break stagnant, and row blood stasis. The tropism of Coptidis Rhizoma (Huanglian) is bitter and cold, in the heart, stomach, liver, large intestine meridians. Coptidis Rhizoma (Huanglian) functions to clear heat and dampness, purge fire and detoxify, and especially clear the heat in heart meridians. The tropism of Scutellariae Radix (Huangqin) is bitter and cold, in the lungs, stomach, gallbladder, large intestine meridians. Scutellariae Radix (Huangqin) functions to clear heat and dampness, purge fire and detoxification, cooling blood and achieving hemostasis, is suitable for clearing lung fire and upper-jiao heat. Coptidis Rhizoma (Huanglian) and Scutellariae Radix (Huangqin) together as ministerial drugs, help Rhei Et Rhizome (Dahuang) "Xie huo". It is consistent with the debate and treatment of TCM that all the drugs work together to clear heat and dampness, relieve fire and detoxify blood. 
Our study screened 42 ingredients and 42 targets of Yiqing capsule in treating COVID-19 based on network pharmacology. The main ingredients were coptisine, naloe-emodin, baicalein, beta-sitosterol, wogonin, Eriodyctiol (flavanone), and oroxylin a. Baicalein and wogonin can reverse lung injury induced by influenza a virus [11]. Baicalein has the same binding energy with the 2019-ncov 3CL hydrolase through molecular docking as does the antiviral drug Remdesivir [12]. Lin, et al. reported beta-sitostero showed an anti-coronavirus effect on blocking the cleavage process of 3CLpro of coronavirus [13].

The results of GO and KEGG analysis shown that the main pathway of Yiqing capsule in treating COVID-19 were GE-RAGE signaling pathway in diabetic complications, IL-17 signaling pathway, Chagas disease (American trypanosomiasis), Influenza A, TNF signaling pathway, and Pertussis. Pathways mainly involve virus infection, inflammation, apoptosis and immune regulation. It is suggested that Yiqing capsule may act on a variety of cytokines to fight inflammation and inhibit cytokine storms in the treatment of COVID-19 [14].

\section{Conclusion}

In summary, the network pharmacology method showed that Yiqing capsule can treat COVID-19 through multiple components, targets and pathways, but it also had some limitations. The tropism of Yiqing capsule is greatly bitter and cold, leading to damage the spleen and stomach injury when taken chronically. Therefore, doctors should limit use of Yiqing capsule to acute treatment. In addition, some of the results in our study still need to be verified by further laboratory tests and clinical studies.

\section{Funding}

This study was funded by the Study and Development Fund for Sciences and Technology in Chengde City (No. 201701A087).

\section{Conflicts of Interest}

The authors declare no conflicts of interest regarding the publication of this paper.

\section{References}

[1] Sohrabi, C., Alsafi, Z., O’Neill, N., et al. (2020) World Health Organization Declares Global Emergency: A Review of the 2019 Novel Coronavirus (COVID-19). International Journal of Surgery, 76, 71-76. https://doi.org/10.1016/j.ijsu.2020.02.034

[2] Ma, J.J., Chen, M. and Wang, Y.G. (2020) Novel Coronavirus (2019-ncov) Syndrome: A Review of TCM Syndrome Treatment. Beijing Journal of Traditional Chinese Medicine, 39, 95-101.

[3] Sun, Z.T., An, X., Xiao, W., et al. (2020) Discussion on Differentiation and Treatment of COVID-19 by Stages. Journal of Shaanxi University of Chinese Medicine. http://kns.cnki.net/kcms/detail/61.1501.R.20200211.1318.002.html

[4] Xu, L.H., Li, Y.R., Zheng, D.R., et al. (2020) To Explore Prescription Law of New 
Coronavirus Pneumonia in Different Stages Based on "Appropriate Measures in Different Time”. Chinese Journal of Experimental Traditional Medical Formulae. http://kns.cnki.net/kcms/detail/11.3495.R.20200225.1145.003.html

[5] Ding, H., Yan, B.H., Tian, L., et al. (2011) A Mutli-Center Randomized Double-Blind Controlled Trial on the Function of Yiqing Capsule Treat. Liaoning Journal of Traditional Chinese Medicine, 38, 1468-1490.

[6] Hou, Y.L., Liu, X.M. and Xu, Q. (2001) Clinical Observation of 156 Cases of Acute Upper Respiratory Tract Infection Treated by Yiqing Capsule and Antibiotics. West China Journal of Pharmaceutical Sciences, 16, 401.

[7] Xu, X.L., Yue, Y., Liu, X.J., et al. (2015) Anti-Virus Effect of Yiqing Capsule on Influenza A Subtype H1N1. The Chinese Journal of Modern Applied Pharmacy, 32, 1056-1058.

[8] Liu, C.H. and Wang, Y. (2020) Discussion on the Application of Febrile Disease Theory to the Diagnosis and Treatment of COVID-19. SH. J. Traditional Chinese Medicine, 54, 5-8.

[9] Zheng, W.K., Zhang, J.H., Yang, F.W., et al. (2020) Comprehensive Analysis of Diagnosis and Treatment Schemes for Prevention and Treatment of Novel Coronavirus Pneumonia by Traditional Chinese Medicine. Journal of Traditional Chinese Medicine, 61, 277-280.

[10] Xu, L.H., Huang, X.J., Li, Y.R., et al. ()2020 Effect and Potential Mechanism of Rhubarb on COVID-19 Based on the "Wenbingzaixiaqiyure" Theory. Pharmacology and Clinics of Chinese Materia Medica. http://kns.cnki.net/kcms/detail/51.1188.R.20200330.1038.002.html

[11] Huang, L.L., Wang, J.N., Xu, R., et al. (2020) Study on Mechanism of HuanglianJiedu Decoction in Treating Novel Coronavirus Pneumonia Based on Network Pharmacology. http://kns.cnki.net/kcms/detail/44.1286.r.20200326.1642.054.htm

[12] Zong, Y., Ding, M.L., Jia, K.K., et al. (2020) Exploring Active Compounds of Da-Yuan-Yin in Treatment of COVID-19 Based on Network Pharmacology and Molecular Docking Method. Chinese Traditional and Herbal Drugs, 51, 836-844.

[13] Lin, C.W., Tsai, F.J., Tsai, C.H., et al. (2005) Anti-SARS Coronavirus 3C-Like Protease Effects of Isatis indigotica Root and Plant-Derived Phenolic Compounds. Antiviral Research, 68, 36-42. https://doi.org/10.1016/j.antiviral.2005.07.002

[14] Liu, R.P., Ge, J.D., Zheng, Q., et al. (2020) Traditional Chinese Medicine for Treatment of COVID-19 Based on Literature Mining of Targeting Cytokine Storm. Chinese Traditional and Herbal Drugs. http://kns.cnki.net/kcms/detail/12.1108.R.20200303.1317.004.html 